\title{
Precision Pulsar Timing in the Presence of Interstellar Weather - PSR B1937+21 and the Crab Pulsar
}

\author{
D. C. Backer and T. Wong \\ Astronomy Department, University of California at Berkeley
}

\begin{abstract}
We report on six months of dual-frequency, precision timing of PSR B1937+21 and the Crab pulsar. Variations in the column density of electrons - interstellar weather - are studied. The column is not unique in the presence of frequency-dependent multipath scattering, and this may explain the non-square law dependence of systematic timing residuals on $\mathrm{B} 1937+21$. The electron column density variations of the Crab pulsar are summarized in a structure function that ranges from $10^{12} \mathrm{~cm}$ to $10^{15} \mathrm{~cm}$. These density fluctuations are located in the thermal shell surrounding the synchrotron nebula.
\end{abstract}

\section{Observations}

We used an $85 \mathrm{ft}$ telescope operated by the National Radio Astronomy Observatory (NRAO) in Green Bank, WV, with funding from the US Naval Observatory and the Naval Research Laboratory (USNO/NRL). Our Coherent Dispersion Removal Processors were used to provide average pulse profiles as a function of frequency channel. The two pulsars are observed for approximately one hour once a day at $327 \mathrm{MHz}$ and twice a day at $610 \mathrm{MHz}$. In offine processing dispersion between channels is removed and the flux scale is linearized and calibrated prior to forming a single pulse profile. Arrival times are estimated by template cross-correlation and are analyzed with a version of the program TEMPO. A copy of the profiles and arrival times will be placed in a public archive that has been started at the USNO (see Matsakis, this conference.)

\section{PSR B1937+21}

The original millisecond pulsar, B1937+21, has been the most precisely timed pulsar (e.g., Kaspi et al. 1994). The millisecond pulsars in general provide a celestial reference frame of accurate clocks that can be employed as a Pulsar Timing Array for studies of a number of fundamental quantities: the stability of our terrestrial atomic time scale; the masses of the outer planets; and the background of gravitational radiation (e.g., Backer 1993). In making timing measurements we must determine the dispersive delay and pulse broadening that occurs as the pulsar signals traverse the turbulent interstellar plasma. Kaspi et al. present a structure function analysis of the delay variations for time intervals from about 2 months to 5 years based on an eight-year span of $1.4 / 2.4-\mathrm{GHz}$ observation from the Arecibo Observatory. Our data allows us to probe both 


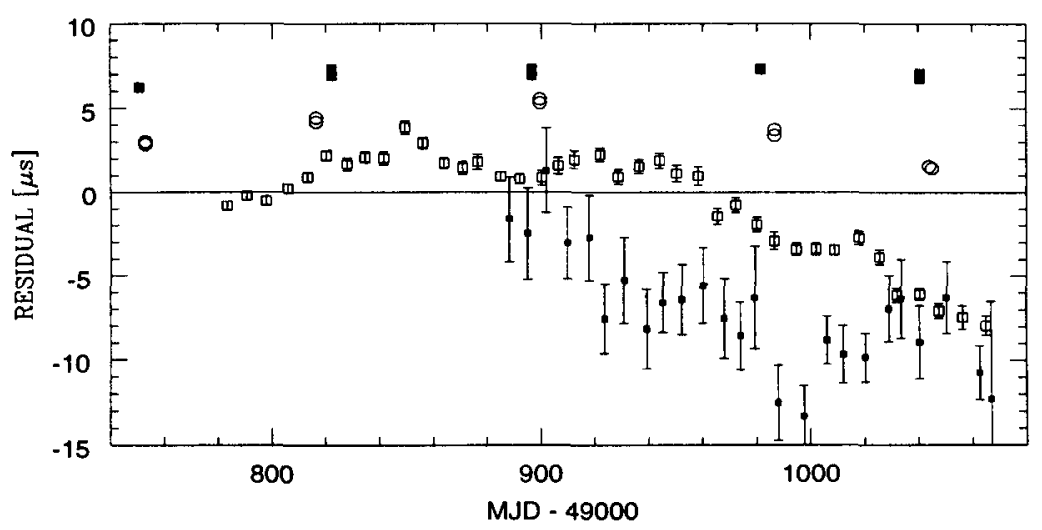

Figure 1. Timing residuals from Nançay 1.4-GHz data model for Green Bank observations at $1.4 \mathrm{GHz}$ (solid square); $0.8 \mathrm{GHz}$ (circle); $610 \mathrm{MHz}$ (open square); $327 \mathrm{MHz}$ (small solid square and error bars). An arbitrary constant has been removed from each set of residuals.

dispersion delay variations on shorter time scales and pulse broadening. In addition we can compare our results with contemporaneous measurements at decimeter wavelengths to confirm the expected inverse square law dependence of the dispersive delay variations on frequency. In this preliminary report we will assume that the pulse broadening effect arising from multipath propagation is not important.

In our data analysis we first fit a model for $P$ and $\dot{P}$ to arrival times kindly provided by $\mathrm{J}-\mathrm{F}$. Lestrade from the Nançay $1.4-\mathrm{GHz}$ timing program. We next apply this model to data obtained bimonthly using the NRAO 140ft telescope at 0.8 and $1.4 \mathrm{GHz}$ and to the $85 \mathrm{ft}$ data at 610 and $327 \mathrm{MHz}$. The day-averaged residuals are shown in Fig. 1. The $1.4-\mathrm{GHz}$ data show negligible residuals, as expected. The $0.8-\mathrm{GHz}$ data show residuals that have a parabolic character over the data set. This is likely the result of dispersive delay changes. The $610-\mathrm{MHz}$ data have residuals that are also parabolic in character with a larger amplitude that is consistent with the interpretation of dispersive delay. However, the 327$\mathrm{MHz}$ residuals are certainly not consistent with a scaled version of the $610-\mathrm{MHz}$ residuals.

We can think of three possibilities to explain the disagreement with simple dispersive delay variations. First, there may be some systematic errors in our signal processing that we have not understood. Second, the $327-\mathrm{MHz}$ data may be affected by variable pulse broadening as a result of interstellar scattering. Variations reported by Ryba (1991) would lead us to conclude that this effect is not important. The third possible explanation is that the volume of plasma sampled at any frequency decreases dramatically with increasing frequency owing to multipath propagation. (Small scale turbulence in the plasma scatters the signal which leads to multipath propagation, pulse broadening and angular broadening.) At any frequency we sample the plasma along a tube that grows in diameter along the path from the pulsar to the midpoint (for a uniform 


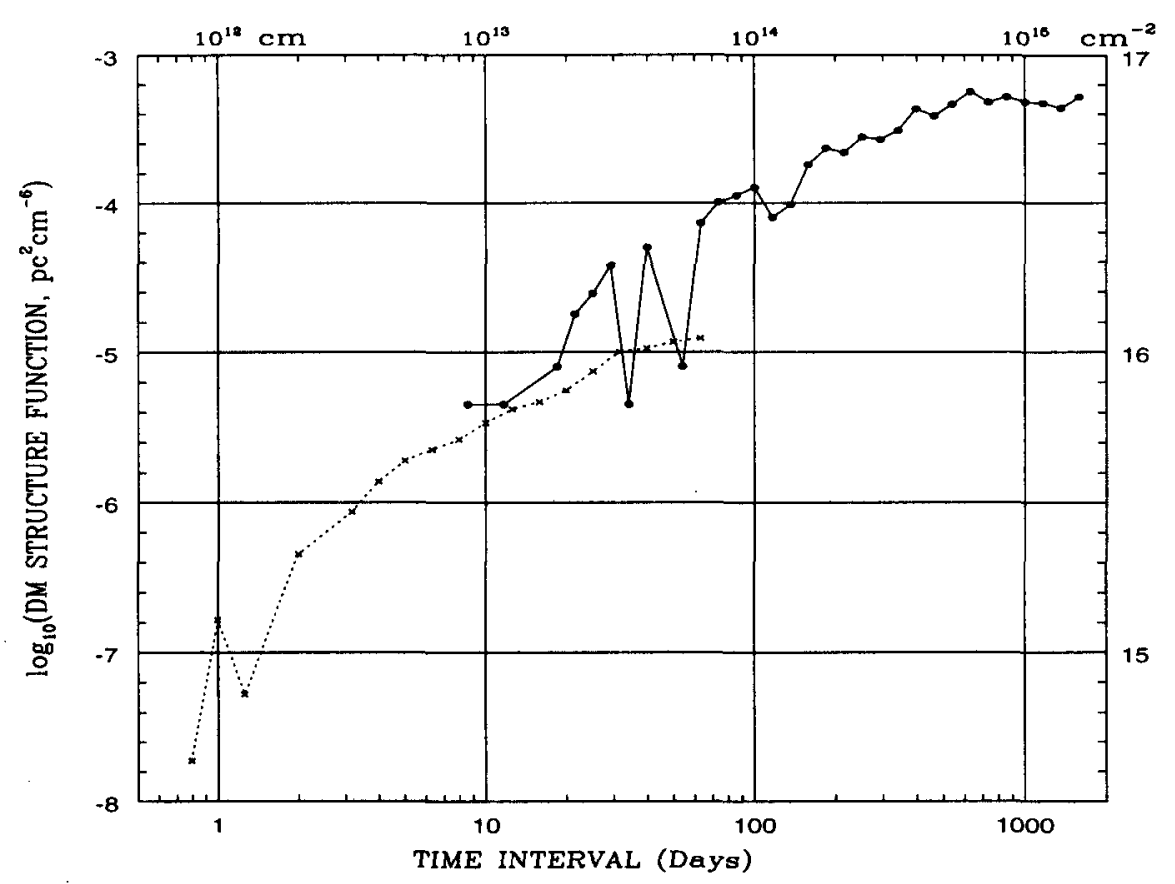

Figure 2. Structure function of dispersion measure from Green Bank (x) and Jodrell Bank (.) observations of Crab pulsar.

medium), and then converges toward the observer. The scattering angle at $\nu=$ $1 \mathrm{GHz}$ is 0.1 mas which leads to a diameter of $10^{13} \mathrm{~cm}$ midway to the pulsar. The scattering angle depends on $\nu^{-2}$. The averaging volume displaces itself on a refractive time scale (e.g., Stinebring contribution, this volume) which is estimated at $10 \nu^{-2}$ days, with $\nu$ in $\mathrm{GHz}$. This model says that the dispersion measure is not uniquely defined for multi-frequency observations. Furthermore dispersion measure variations will be suppressed on time scales less than the refractive time scale if the medium is frozen. We plan to test this model closely with this data set.

\section{Crab Pulsar}

The $327-\mathrm{MHz}$ and $610-\mathrm{MHz}$ timing of the Crab pulsar reveals two phenomena: frequency independent timing noise which was studied intensively by Groth $e t$ al. (1975) and other authors, and dispersion measure variations which were studied by Rankin et al. (1988). In this discussion we again assume that the time-variable effects of interstellar scattering are negligible. (In more recent data scattering has increased dramatically.) The dispersion measure variations are obtained from the difference in the arrival time residuals at the two frequencies, with the measurements analyzed using the identical timing model. The disper- 
sion measure deviations are then used to correct the $610 \mathrm{MHz}$ data to infinite frequency to explore the short term statistics of timing noise. We will not report on timing noise in this brief discussion.

The dispersion measure variations have been analyzed to form a structure function that extends over time intervals from 1 to 50 days. In Figure 2 we compare our structure function with that obtained from the Jodrell Bank data (courtesy of A. Lyne) which samples time intervals from 50 to 1000 days. The joined structure function has a logarithmic slope of about -1 for short time intervals. The structure function flattens beyond a time interval of about 700 days. With a 13-year record available, this turnover may be a significant and we can infer the presence of a saturation scale.

The rapid variations in dispersion measure are very likely the result of density fluctuations in the envelope of ionized gas that surrounds the Crab synchrotron nebula and contains the optical line emitting filaments (Lawrence et al. 1995; Hester et al. 1996). This was the early conclusion of Rankin et al. (1988) and was supported by a summary of the level of dispersion measure variations from the general interstellar medium by Backer et al. (1993). Assuming this location for the turbulent gas we can convert the structure function in time interval to one in transverse length. Asymmetries in the radially expanding gas motion and the pulsar proper motion lead to an estimate of $200 \mathrm{~km} \mathrm{~s}^{-1}$ for the transverse velocity of the gas. The 1-1000 day structure function then samples transverse length scales of $2 \times 10^{12} \mathrm{~cm}$ to $2 \times 10^{15} \mathrm{~cm}$. The high resolution imaging of the filamentary gas obtained by HST (Hester et al. 1996) will allow us to sample the structure function of the ionized gas density variations in the vicinity of the pulsar over transverse length scales from $10^{16} \mathrm{~cm}$ to $10^{18} \mathrm{~cm}$. The relation between the amplitudes of the column density and emission measure structure functions from these two techniques will be uncertain owing to unknown filling factors, but the slopes may be compared.

Acknowledgments. We thank the USNO, NRL and NRAO for use of the $85 \mathrm{ft}$ telescope. We also thank D. Stinebring, D. Nice, and their collaborators for initiating and maintaining the pulsar use of this telescope. A. Zepka helped in the preparation of Figure 2. This work was partially supported by NSF Grant AST-93-07913.

\section{References}

Backer, D. C. 1993, in Proc. 4th Predoctoral Summer School of EADN, ed. J. van Paradis, [Springer : Berlin] LNP 418, 193.

Backer, D. C., Hama, S., Van Hook, S., \& Foster, R. S. 1993, ApJ, 404, 636.

Groth, E. J. et al. 1975, ApJS, 29, 453.

Hester, J. J. et al. 1996, ApJ, in press.

Kaspi, V. Ḿ. et al. 1994, ApJ, 428, 713.

Lawrence, S. S. et al. 1995, AJ, 1092635.

Rankin, J, M. et al. 1988, A\&A, 202, 166.

Ryba, M. F. 1991, Ph. D. Thesis, Princeton University. 